Cahiers « Mondes anciens »

ANCIENS

Histoire et anthropologie des mondes anciens

$10 \mid 2018$

Les politiques familiales dans les mondes antiques

\title{
Un toit, des lois
}

Les politiques familiales dans les mondes anciens

Law at Home. Family Policy in Ancient Worlds

Sabine Armani et Aurélie Damet

\section{(2) OpenEdition}

Journals

Édition électronique

URL : http://journals.openedition.org/mondesanciens/2059

DOI : 10.4000/mondesanciens.2059

ISSN : 2107-0199

Éditeur

UMR 8210 Anthropologie et Histoire des Mondes Antiques

Référence électronique

Sabine Armani et Aurélie Damet, « Un toit, des lois », Cahiers « Mondes anciens » [En ligne], 10 | 2018,

mis en ligne le 13 mars 2018, consulté le 02 mai 2019. URL : http://journals.openedition.org/

mondesanciens/2059; DOI : 10.4000/mondesanciens.2059

Ce document a été généré automatiquement le 2 mai 2019.

\section{c) (1) (9)}

Les Cahiers «Mondes Anciens » sont mis à disposition selon les termes de la licence Creative Commons Attribution - Pas d'Utilisation Commerciale - Pas de Modification 4.0 International. 


\section{Un toit, des lois}

Les politiques familiales dans les mondes anciens

Law at Home. Family Policy in Ancient Worlds

Sabine Armani et Aurélie Damet

\section{Politiques publiques et politiques familiales}

1 Une politique familiale est aujourd'hui définie comme l'ensemble des mesures et des interventions publiques, gouvernementales ou municipales, destinées aux familles: la politique familiale "est le résultat de l'interaction de plusieurs champs d'activité ", à savoir le champ politique, le champ juridique et son droit de la famille, mais aussi le champ médical (Lenoir 2007). Les sociologues, spécialistes des politiques publiques, comme Jean-Claude Thoenig (2014), et des politiques sociales familiales, comme Claude Martin (2011), insistent sur la spécificité française en terme d'intervention de l'État dans la question familiale. C'est avant tout au lendemain de la Seconde Guerre Mondiale que s'est mise en place une série de mesures étatiques concernant les familles et la famille, des lois influencées parfois par des courants de pensée, médicale, hygiéniste ou démographique, qui font ainsi partie intégrante de l'étude des politiques publiques envers la famille : les législateurs agissent dans un cadre qui n'est pas seulement juridique et législatif, mais aussi culturel ${ }^{1}$. En l'espace de plusieurs décades, la France a ainsi vu la mise en place de privilèges pour les familles nombreuses, la dépénalisation de l'avortement et l'autorisation de la contraception, la réforme du divorce et de l'autorité parentale, la mise en place des congés parentaux et de l'aide à la garde d'enfants, le PACS, ou encore l'égalisation récente des droits à l'héritage des enfants dits adultérins ${ }^{2}$. De très nombreuses décisions ont alors façonné la famille française et les rapports intrafamiliaux depuis 1945.

2 Pour analyser ces politiques familiales, les sociologues ont recours à deux types d'opposition :

- «L'universalité » contre « la sélectivité »: une politique familiale peut ainsi concerner ou bien toutes les familles ou seulement quelques familles, choisies le plus souvent 
d'après des critères socio-économiques.

- «Le familialisme» contre "l'individualisme»: les politiques familiales concernent toute la famille, ciblée en bloc, ou bien elles privilégient des individus à l'intérieur du cercle familial, les plus vulnérables, comme les familles monoparentales, les enfants, ou encore les personnes âgées, considérés ainsi comme des "catégories institutionnelles » (Roubineau 2012, p. 166).

3 Ce tableau, très résumé ici, de la politique familiale française donne un cadre conceptuel intéressant dans lequel on peut penser les rapports entre la famille et le pouvoir dans les mondes anciens, thématique au cœur de ce volume d'articles. Si, dans le Proche-Orient ancien, Sophie Démare-Lafont conclut, dans sa contribution, que "la vieillesse, l'isolement, la précarité, ne sont pas pris en charge par l'État mais par les proches ou la collectivité", la Grèce archaïque et classique semble offrir davantage de sources témoignant d'un vrai souci des autorités civiques de protéger et de modeler la famille.

\section{Unions permises et prohibées, divorces et adultères : les politiques matrimoniales des législateurs grecs}

4 L'un des domaines d'intervention des législateurs en matière d'organisation familiale est la mise en place de politiques matrimoniales, qui orientent nettement le choix des conjoints et réglementent les modalités de rupture d'alliance. Ainsi que le rappelle Françoise Ruzé (2006, p. 174), dans son étude sur les législations archaïques et l'aide aux plus faibles :

Les lois se soucient de la protection du couple, en poussant à la fidélité conjugale, en réglementant le divorce et en surveillant les femmes. À Athènes, Solon aurait donné le cadre du mariage "légitime", celui qui permet d'avoir des enfants légitimes: il suppose que la fille ait été confiée selon les règles ainsi établies, par son plus proche parent mâle, à l'homme qui deviendra son mari et son kyrios, le temps du mariage ; ainsi, sans jamais maitriser sa destinée, la femme passe d'une autorité à l'autre. Désormais, la cellule familiale étant légalement circonscrite, c'est sur le couple ainsi formé que la cité légifèrera.

5 Jérôme Wilgaux revient, dans sa contribution, sur la loi de Périclès de 451, qu'il replace dans une tendance contextuelle à la fermeture des groupes matrimoniaux, comme par exemple en Judée. À Athènes, la loi de Périclès est mise en place au moment où se pose la question de circonscrire le groupe des bénéficiaires des retombées de l'impérialisme. L'arrière-plan est certes économique mais il est aussi idéologique: désormais, les metroxenoi, les «sang-mêlé », sont exclus de la citoyenneté, alors que se popularise la thématique autochtone ${ }^{3}$.

6 Touchant à la question matrimoniale, la législation sur l'adultère est aussi une facette des politiques de la famille; il s'agit avant tout d'éviter la naissance d'enfants illégitimes. Si le dossier athénien à ce propos est bien connu (procédure de la graphê moicheias, meurtre légal de l'amant par le mari cocu en cas de flagrant délit, tortures infamantes, atimie cultuelle et répudiation obligatoire de l'épouse adultère) ${ }^{4}$, Diodore de Sicile apporte un précieux témoignage sur les législateurs archaïques de Grande Grèce, Zaleucos de Locres et Charondas de Catane (Ruzé 2006). On trouve chez les deux hommes un souci partagé de stigmatiser les coupables d'adultère et, en creux, d'en éviter la prolifération. Selon Stobée (Florilège, IV, 2, 24), Charondas de Catane aurait ainsi édicté : 
Que chacun aime sa femme légitime et fasse des enfants avec elle, mais qu'il n'aille pas répandre ailleurs la semence de ses enfants; qu'il n'aille pas dépenser sans respect des lois et dans l'excès ce que la nature et la loi lui ont donné de précieux. En effet, la nature a donné la semence pour la procréation et non pour la licence. Il faut que la femme soit sage et qu'elle évite toute relation impie avec d'autres hommes, car elle rencontrerait la vengeance des démons qui chassent de la maison et qui créent l'hostilité (trad. Ruzé 2006).

7 Zaleucos de Locres, qui partagea « les mêmes règles de vie » que Charondas (Diodore de Sicile, XII, 19, 3) légiféra ainsi sur l'adultère :

Ainsi, tous les autres n'avaient infligé que des amendes aux femmes coupables, mais lui contint leurs débordements par un châtiment plein d'astuce. Voici ses prescriptions: une femme libre ne devait pas être accompagnée de plus d'une servante, à moins qu'elle ne fût ivre, ni sortir de la ville la nuit, à moins qu'elle n'eût un amant, ni se parer de bijoux d'or ou d'un vêtement bordé de pourpre, à moins qu'elle ne fût prostituée... (Diodore de Sicile, XII, 21, 1).

Zaleucos, selon Héracleidès (FHG, II, p. 221), aurait ainsi précisé le sort des hommes adultères :

Si quelqu'un est pris en flagrant délit d'adultère, on lui arrache les yeux. Le fils de Zaleucos fut pris ainsi mais les Locriens l'acquittèrent ; Zaleucos ne l'accepta pas et arracha l'un de ses yeux à lui, et un autre à son fils (trad. Ruzé 2006).

Outre les unions et les adultères, les réglementations sur le divorce se mettent aussi en place et encadrent les ruptures d'alliances, en stipulant parfois les conditions de séparation et les droits des époux ${ }^{5}$.

Sparte est aussi une cité où les lois, pour la plupart rattachées au législateur semi-légendaire Lycurgue, ont largement modelé les relations intrafamiliales et l'organisation des maisons. D'après les témoignages de Critias, Xénophon ou encore Plutarque, les arrangements matrimoniaux valorisaient les meilleurs métabolismes, qui combinaient des qualités de naissance et des qualités acquises par des mœurs et un mode de vie façonnant les meilleurs corps. Aurélie Damet revient dans ce volume sur cet eugénisme spartiate, distillé notamment dans les pratiques matrimoniales dites «polyandriques». L'idéologie eugénique n'est pas une exception lacédémonienne et les utopies politiques ont largement utilisé le principe de hiérarchie et de sélection des meilleurs reproducteurs (Damet 2017). D'une manière générale, la République et les Lois de Platon, et les Politiques d'Aristote, sont truffées de mesures législatives qui couvrent de nombreux domaines en lien direct avec la famille: politique nataliste, politique eugénique, politique matrimoniale, politique patrimoniale ou encore réflexions démographiques. Ces dernières soulignent notamment le cas particulier de la société spartiate, frappée du phénomène d'oliganthropie (Aristote, Politiques, 1270a). C'est dans le cadre de la lutte contre ce fléau que l'on peut resituer les législations existantes sur la lutte contre le célibat, ce dont l'article de Florence Gherchanoc rend compte dans ce volume. L'arbre du célibat cache bien évidemment la forêt de la dénatalité : encourager les unions, les mariages et donc les naissances est aussi un champ d'action privilégié des politiques familiales.

\section{Encourager les naissances}

$11 \mathrm{Au} \mathrm{II}{ }^{\mathrm{e}}$ siècle av. J.-C., l'auteur Polybe se lamente ainsi sur l'état de dépeuplement de sa patrie d'origine, la Grèce : 
De nos jours, dans la Grèce entière, la natalité est tombée à un niveau très bas et la population a beaucoup diminué, en sorte que les villes se sont vidées et que les terres restent en friche, bien qu'il n'y ait pas eu de longues guerres ni d'épidémies. Si quelqu'un s'avisait d'envoyer aux dieux ce qu'il faudrait dire ou faire pour que nous nous retrouvions plus nombreux et pour que nos villes soient plus peuplées, cette démarche ne semblerait-elle pas absurde puisque la cause du mal est évidente et qu'il dépend de nous d'y remédier? Les gens de ce pays ont cédé à la vanité et à l'amour des biens matériels; ils ont pris goût à la vie facile et ils ne veulent plus se marier ou, quand ils le font, ils refusent de garder les enfants qui leur naissent ou n'en élèvent tout au plus qu'un ou deux, afin de pouvoir les gâter durant leur jeune âge et de leur laisser ensuite une fortune importante. Voilà pourquoi le mal s'est, sans qu'on s'en fût rendu compte, rapidement développé. En effet, quand il n'y a qu'un ou deux enfants, il suffit que la guerre en enlève un et la maladie un autre pour que les foyers, inévitablement, se vident. Alors, tout comme les essaims d'abeilles, les cités, elles aussi, se vident de leur substance et s'étiolent peu à peu. Il n'est pas nécessaire d'interroger les dieux pour savoir comment nous pourrions nous délivrer de ce fléau. Le premier venu nous dira que cela dépend avant tout de nous et que nous n'avons qu'à nourrir d'autres ambitions, ou qu'à défaut nous devrions faire passer des lois obligeant les parents à élever leurs enfants (Polybe, Histoires, XXXVI, 17, 5-10).

12 Contre le fléau oliganthropique, Polybe en arrive ainsi à souhaiter que les instances dirigeantes des cités instaurent des lois, qu'on appellerait aujourd'hui natalistes: ces mesures auraient pour finalité d'obliger les individus à se marier et à élever les enfants qui leur naîtraient, ce qui induirait d'ailleurs une limitation du droit à l'exposition. Si l'on revient dans l'Athènes classique, Périclès, dans sa fameuse oraison funèbre, incitait bien les Athéniens à faire des enfants et à remplacer les fils morts ${ }^{6}$. Selon Aristote (Politiques, 1270b) la cité spartiate aurait mis en place de telles incitations à la procréation, en exemptant de service militaire les pères de familles nombreuses : «Car en voulant que les Spartiates soient les plus nombreux possible, le législateur incite les citoyens à faire le plus d'enfants possibles : il y a, en effet, une loi qui dispense le père de trois fils de service militaire et celui de quatre fils de toute imposition. » Les fils étaient utiles à la cité et à sa défense; et ils étaient aussi utiles à leurs parents, dans des sociétés où l'aide aux personnes âgées dépendait du principe de solidarité intergénérationnelle, cependant étayée par la loi.

\section{L'encadrement légal de la solidarité intergénérationnelle : paidotrophia et gêrotrophia}

13 Le code de Gortyne est une source précieuse pour tout historien de la famille : héritage, adoption, exposition des enfants, solidarité intergénérationnelle, divorce, fille-héritières... La thématique familiale irrigue la quasi-totalité du document crétois. C'est précisément la question des obligations des descendants, dans le code de Gortyne, que Maude Lajeunesse a choisie pour objet d'étude dans ce volume. Dans l'Athènes classique, les mesures qui ciblent là encore l'organisation de l'oikos et de la parenté des droits et des devoirs, l'anchisteia, sont nombreuses. La marque juridique de l'intérêt de la polis athénienne pour les populations âgées est notamment l'existence d'une procédure publique (une graphê), que tout citoyen peut intenter à un autre citoyen, s'il le soupçonne de mauvais traitements (Damet 2012). La graphê kakôseôs goneôn couvre ainsi les diverses offenses dont des parents pourraient être victimes : le refus de nourriture (Eschine, Contre Timarque, 13 ; Démosthène, Contre Timocrate, 107) et de logement (Eschine, Contre Timarque 
13), mais aussi les coups portés (Lysias, Contre Agoratos, 91), et le non-respect des devoirs funéraires (Xénophon, Mémorables, II, 2, 13 ; Lycurgue, Conte Léocrate, 147). L'obligation de nourriture était pensée sur le mode du don/contre don : à la paidotrophia répondait la gêrotrophia. Mais elles dépassaient toutes deux les frontières de l'entraide familiale informelle, en étant l'objet d'un strict encadrement légal. Démosthène évoque d'ailleurs les «lois protectrices de la vieillesse " ( tous tô gêra boèthous nomous", Contre Timocrate, 107). En miroir, Eschine (Contre Timarque, 13) et Plutarque (Vie de Solon, 22, 1) rappellent les cas où le père défaillant ne peut attendre de la part de son fils une aide durant ses vieux jours, s'il l'a livré à la prostitution ou s'il ne l'a pas éduqué en vue de l'apprentissage d'un métier. Delfim Leao reprend ici ce dossier de l'encadrement légal de la paidotrophia et de la gêrotrophia, en privilégiant, pour source documentaire, l'Alceste d'Euripide, dont le scénario repose sur l'affrontement entre un fils et ses parents.

\section{La protection des orphelins et des épiclères dans les cités grecques}

La protection des orphelins est bien attestée dans le monde grec ancien, notamment la prise en charge des orphelins de guerre, à Athènes, Thasos ou encore Rhodes ${ }^{7}$ et, d'après Aristote, elle constituait un pan courant des législations ${ }^{8}$. Cette politique de protection est un bon exemple de la tension entre " universalisme » et "sélectivisme ", ainsi qu'entre « familialisme » et « individualisme ». À Athènes, lors de la restauration démocratique de 403, dans un contexte de compression nécessaire des dépenses publiques, Théozotidès aurait fait modifier les modalités d'octroi de l'aide de l'État athénien aux enfants dont le père est mort à la guerre, apportant deux amendements (Stroud 1971; Slater 1993; Fournier et Hamon 2007 ; Roubineau 2012). Tout d'abord, le décret de Théozotidès élargit aux enfants des victimes des Trente Tyrans les bénéficiaires, assimilant ici polemos et stasis. Mais, il restreint cependant les privilèges matériels prévus aux enfants biologiques reconnus, les gnêsioi, en excluant donc les enfants adoptés et les enfants «nothoi ». On a conservé ainsi un court fragment de Lysias, qui s'insurge contre la discrimination induite par cet amendement, et qui considère que, justement, ce sont bien les enfants nothoi, exclus de l'héritage à partir de 403, qui sont dans le besoin, beaucoup plus que les gnêsioi qui, eux, récupèrent le patrimoine paternel. Mais, vraisemblablement, la cité ne pouvait pas être nourricière pour tous ces (ses) enfants laissés sans père 9 .

Par cette loi [...] mais surtout [...] témoignant, à l'égard des fils naturels (nothous) et des fils adoptifs (poiêtous), d'une haine qui est contraire à la loi et à la religion. Je trouve, quant à moi, que, parmi les orphelins, les fils naturels, plus encore que les fils légitimes, ont le droit d'être entretenus par la cité : car ceux-ci, leur père, en mourant, les laisse héritiers de son bien, tandis que les fils naturels [...] pour la solde [...] il leur a laissé [...] Mais, le plus révoltant, c'est que, grâce à Théozotidès, la plus belle des proclamations que les lois ordonnent sera décriée et taxée de mensonge. Lors des Dionysies, lorsque le héraut annoncera, en appelant les orphelins par le nom de leurs pères: "voici les jeunes gens dont les pères sont morts en combattant bravement pour la patrie, et que la cité a élevés jusqu'à leur majorité ", fera-t-il une annonce spéciale pour les fils adoptifs et naturels en disant : « ceux-là, sur la proposition de Théozotidès, la cité ne les a pas élevés » ? Ou bien, faisant une proclamation commune à tous les orphelins, mentira-t-il sur le compte des enfants adoptifs et naturels, en se taisant sur leur éducation ? Quel outrage pour la cité, et quel discrédit ! (Lysias, Contre Théozotidès, 1-2). 
15 Lysias plaide pour ce qu'on appellerait aujourd'hui une «socialisation de la politique familiale ", qui prenne en compte le statut économique des lésés de la loi. La question de la politique familiale croise ainsi celle des politiques sociales, entendues comme des mesures visant à remédier aux difficultés économiques de certaines populations. Dans l'Athènes classique, les nothoi avaient conservé jusqu'en 403 une vocation héréditaire en l'absence de fils légitimes. Le décret d'Aristophon avait cependant, au moment de la restauration démocratique qui suit la tyrannie des Trente, réinstauré une distinction nette entre notheia et gnêsiotês ${ }^{10}$. L'amendement de Théozotidès allait ainsi dans le même sens d'une discrimination des nothoi, mais aussi des poiêtoi, dont l'exclusion des bénéfices d'État est ici intrigante. Les sources de l'Athènes classique présentent, il est vrai, cette double facette de l'adoption, pratique assez courante, mais hiérarchiquement inférieure à l'engendrement biologique, kata phusin (Damet 2012, p. 151-160). On notera cependant que le schéma familial d'un parent adoptant laissant un enfant adopté mineur à sa mort est probablement peu fréquent : l'adopté était, le plus souvent, un jeune adulte en âge de prendre en charge l'oikos de son père social et de s'occuper de lui pendant ses vieux jours (Rubinstein 1993). Niall Slater (1993) a proposé une interprétation intéressante à ce propos. Si le décret de Théozotidès exclut les poiêtoi, c'est qu'il y aurait eu de nombreux abus pendant la tyrannie des Trente, précisément en matière d'adoption. En effet, les Trente tyrans auraient réformé la loi de Solon qui limitait les possibilités d'adoption si l'adoptant/testataire était sénile, sous l'emprise de la folie ou sous l'influence d'une femme, et ce dans le but de désengorger les tribunaux, saisis trop souvent sur la base de cette clause restrictive ${ }^{11}$. Mais, ce faisant, les Trente auraient permis la multiplication des adoptions posthumes, permettant ainsi de faire bénéficier les enfants adoptés des privilèges des orphelins (panoplie et allocation de subsistance). On voit cependant que la loi de Solon est de nouveau en vigueur au $\mathrm{IV}^{\mathrm{e}}$ siècle et demeure la base de biens des différends dans les tribunaux attiques : le plaidant de la Succession de Ménéklès insiste par exemple sur le fait que Ménéklès n'était pas sénile ni sous l'influence de son ancienne épouse lorsqu'il décida de l'adopter.

16 Si les Athéniens n'ont pas inclus le paramètre socio-économique dans le traitement des orphelins de guerre, les Thasiens ont, eux, réservé aux plus nécessiteux le privilège de la trophê (jusqu'à quatre oboles par jour, beaucoup plus que l'unique obole athénienne), tout en offrant, à tous les orphelins de guerre, une panoplie et le privilège de proédrie, ainsi qu'une invitation à banqueter lors du repas sacrificiel en l'honneur des "Braves" (Hamon et Fournier 2007). Les enfants de métèques et les enfants nothoi recevaient aussi une indemnité forfaitaire, inférieure au montant octroyé aux fils de citoyens, dès lors que leur père était mort pour la patrie. Ainsi, contrairement au décret thasien, le décret de Théozotidès aurait été plus honorifique que social, en ne distinguant pas les bénéficiaires selon les classes censitaires existantes.

17 J.-M. Roubineau (2012), dans un article consacré à la question de l'action sociale à Athènes, conclut à ce propos que «la pauvreté dans les cités n'est pas l'objet d'un traitement spécifique», et rejette ainsi l'existence de politiques sociales. En ceci, il concède ne pas partager, par exemple, l'analyse de J. Ouhlen (2004, p. 348), qui écrit, à propos des misthoi : « destinés à tous les citoyens - pauvres et riches - les subsides publics ne peuvent être interprétés comme une forme d'assistance publique destinée à entretenir une foule de nécessiteux. Seule l'aide financière octroyée aux invalides (Lys. 24), aux orphelins de guerre - fille (dot) et garçons (bouclier) -, ou aux filles thètes épiclères (dot Dém. 43.54), peut être strictement décrite comme une aide sociale». On pourrait aussi 
objecter à J.-M. Roubineau que, certes, les orphelins de guerre sont avant tout privilégiés du fait de leur "statut patriotique ", mais que l'existence de procédures publiques (graphê , eisangélie, phasis), concernant l'ensemble des orphelins, prouve bien que cette catégorie d'enfants était circonscrite comme potentiellement en danger. À Athènes, la tutelle des orphelins revêtait un caractère mixte : il existait non seulement des tuteurs individuels de chaque enfant, parents proches, mais aussi une instance publique de surveillance de tous les orphelins, en la personne de l'archonte-éponyme ${ }^{12}$. Ce dernier pouvait infliger une amende à hauteur de cinquante drachmes contre les personnes qui le gênaient dans l'exercice de ses fonctions ${ }^{13}$. Le fait que l'autorité publique de l'archonte éponyme soit en charge de la sécurité des mineurs montre la présence de l'état dans les affaires des plus faibles, et la volonté de prévenir tout différend intrafamilial. Les vélléités de porter atteinte à la personne d'un orphelin devaient être réfrénées par l'existence de cette instance étatique, qu'é. Karabélias (2002), reprenant l'expression de L. Gernet, qualifie d'« office de ministère public » ${ }^{14}$. De même, si les jeunes filles orphelines sont dotées par les cités de Rhodes et de Thasos ${ }^{15}$, lorsque le père est mort au combat, la catégorie générique des épiclères est plus largement protégée par les lois de la cité athénienne : tout comme il existait une graphê pour mauvais traitement envers les parents ou les orphelins, il existait une graphê couvrant les offenses faites aux épiclères (Damet 2012, p. 225-235). Par ailleurs, à propos de la loi obligeant les ayants droit de l'anchisteia athénienne, en présence d'une épiclère pauvre, à l'épouser ou de la doter (Démosthène, Contre Macartatos, 54), J.-M. Roubineau admet bien qu'« en l'absence d'intérêt financier, l'ensemble du système aurait, à défaut de loi, reposé sur le sens moral des ayants droit, base bien fragile. La loi vient corriger ce risque de dysfonctionnement». J. Roy (1999) insiste aussi sur la nécessité de ne pas surestimer l'efficacité réelle de la solidarité parentale: l'aide intrafamiliale et intergénérationnelle doit ainsi être perçue comme étant à la fois auto-générée par les liens de parenté et suscitée par l'encadrement légal. Évelyne Scheid, dans ce volume, reprend le dossier épicléral, et conclut ainsi que « La cité apparait soucieuse de prendre en compte la protection des filles de citoyens non encore mariées et devenues épiclères du fait du décès de leur père ». L'intérêt de la cité est aussi politique et démographique : il s'agit de favoriser le mariage des épiclères citoyennes afin qu'elles puissent perpétuer l'oikos et, par la-même, perpétuer le corps civique ${ }^{16}$.

18 Dans une étude sur le principe de minorité dans le droit athénien, S. Adam-Magnissali (2007, p. 156) évoquait déjà « la protection légale de l'enfance » et admettait qu'« il existe certaines données qui nous permettent de penser que le législateur athénien reconnaissait implicitement un état d'immaturité des personnes de la petite enfance et s'efforçait, selon des modalités diverses, de les protéger ». Les intérêts de la cité et de l' oikos, à savoir la préservation des patrimoines, croisaient aussi ceux des populations fragiles circonscrites comme telles. Les enfants étaient aussi, selon S. Adam-Magnissali, bénéficiaires d'un "droit à l'alimentation et à l'éducation ». L'enfance et la jeunesse faisaient ainsi l'objet d'une " prévoyance légale » (p. 158). C'est encore sous l'angle d'une politique de protection des orphelins que Diodore de Sicile analyse certaines dispositions prises par Charondas de Catane :

Charondas rédigea encore une autre loi estimable, la loi sur la protection des orphelins. À la considérer superficiellement, elle semble n'avoir rien d'admirable ou d'estimable, mais, après un examen approfondi et détaillé, on découvre sa sagesse et sa valeur très grandes. Elle confie la gestion de la fortune des orphelins aux plus proches parents en ligne paternelle, leur éducation aux parents utérins. De fait, à première vue, rien de sage ou d'admirable dans cette loi, mais on n'a qu'à 
l'examiner à fond pour trouver qu'elle mérite de justes louanges. Cherchez pourquoi le législateur a chargé ceux-là de gérer la fortune des orphelins, ceux-ci de les éduquer, vous y verrez la preuve admirable de sa sagesse : les parents utérins, n'ayant rien à faire avec l'héritage, n'attenteront pas à la vie de leur pupille; les parents consanguins ne le peuvent pas non plus, puisque la personne de l'orphelin ne leur est pas confiée; comme d'autre part, sa fortune leur revient s'il meurt victime d'une maladie ou d'accident, ils gèreront plus soigneusement ses biens qu'ils espèrent, le hasard aidant, hériter (XII, 15, 1-3).

Rapprochant la législation de Charondas des dispositions du Code de Gortyne ${ }^{17}$, F. Ruzé (2006) conclut que, «dans les deux cas, à Catane comme à Gortyne, c'est la même préoccupation qui se manifeste : méfiance envers la rapacité des familles, garanties pour les héritiers trop jeunes ou trop faibles de se défendre eux-mêmes ». Il en ressort finalement que, dans les cités grecques antiques, tout comme dans la France actuelle, on peut analyser « la famille comme cause nationale, comme institution, puis comme partie de la politique sociale » (Lenoir 2007, p. 10). Qu'en est-il des lois romaines?

\section{Rome et la famille}

Du côté de Rome, on se heurte dans un premier temps à un déficit de vocabulaire pour parler de "politique familiale ». Les sources n'offrent aucun parallèle à ces expressions. L'adjectif même de «familial» pose en soi une question méthodologique puisque la famille contemporaine ne correspond pas à la conception romaine de la familia ${ }^{18}$ qui désigne tout le patrimoine du paterfamilias : sa femme épousée sous le régime de la manus, ses enfants non émancipés, les petits-enfants de ces mêmes fils, ses affranchis et ses esclaves. Pour R. P. Saller (1984), le terme qui se rapprocherait le plus de celui de la famille telle que nous la pratiquons est celui de domus. Le mot introduit cependant une nuance qui caractérise davantage les parentèles médiévales et modernes, souvent désignées d'ailleurs comme des structures à «maison » qui vivent sous le même toit, et admet mieux la traduction de "maisonnée ${ }^{19}$ ». Peut-on pour autant aller jusqu'à dire que les autorités - que ce soit les magistrats ou l'empereur - n'ont jamais légiféré pour réguler les relations entre les necessarii (parents), propinqui (les proches), les sui (les siens) qui, tout compte fait, sont les termes les plus appropriés pour désigner la famille telle qu'on la conçoit aujourd'hui, c'est-à-dire le cercle le plus serré de la parenté cognatique.

Il n'est pas question de nier qu'à Rome, la famille constitue le pilier de la société. Il suffit de relire Cicéron pour s'en persuader. Dans le premier livre du de Officiis (I, 54, 5-6), il affirme que " étant donné, en effet ce trait de la nature, commun aux êtres vivants, qu'ils ont le désir d'engendrer, la société réside d'abord dans le couple conjugal lui-même, puis dans les enfants ; ensuite, c'est une seule maison et toutes choses communes. C'est cela le principe de la cité et comme la pépinière de la république ». Cependant, le législateur n'intervient pas dans tous les champs de la vie familiale pour la bonne raison qu'à Rome, la puissance paternelle dont Gaius (Institutes, I, 55) écrivait qu'elle était l'apanage des Romains ${ }^{20}$, constituait l'épine dorsale d'une bonne partie des relations familiales. Si l'on reprend les catégories actuelles qui définissent ce qui constitue grosso modo une politique familiale, force est de constater que l'on peut toujours reconnaître l'une d'elles à l'œuvre dans telle loi romaine ou tel rescrit impérial : ainsi, le «familialisme » qui consiste à défendre un certain type de famille peut être identifié dans la politique "nataliste " d'Auguste tandis que les tenants de la "sélectivité » liront sa mise en œuvre dans le traitement inégal entre familles ingénues et d'origine servile. On peut reconnaître une 
certaine forme de l'« universalité » - qui inspira la politique sociale de l'après-guerre en France - dans les distributions alimentaires qui bénéficiaient à une bonne partie des membres de la plèbe urbaine tirés au sort. En revanche, l'« individualisme » qui consiste à privilégier certains membres de la famille à d'autres, se déduira de l'absence, dans la désignation d'ayants droit, des représentants du sexe féminin dans l'assistance alimentaire $^{21}$.

L'espace laissé à l'historien de l'Antiquité pour évoquer le sujet des "politiques familiales à Rome » est donc forcément étroit et limité. Pourtant, il serait erroné de penser qu'à leur manière, les législateurs romains ne sont pas intervenus en matière de droit de la famille. Il est bien sûr inutile d'essayer de trouver des équivalents à nos systèmes contemporains d'aides à la famille, ne serait-ce que parce que l'un des critères qui nous semble le plus juste aujourd'hui et qui oriente, notamment en temps de crise, les évolutions des politiques familiales - celui de la pauvreté - laissa indifférent le législateur romain. Il n'y a donc pas de "politique familiale » institutionnalisée, mais des formes d'intervention du pouvoir destinées à réguler certains aspects de la vie quotidienne et qui touchent de près ou de loin à la famille. Trois domaines ont particulièrement retenu l'attention des autorités : la question des statuts des individus et la manière dont ils peuvent ou non s'allier, celle de la transmission (du nom, des biens) et la bonne marche de la cité.

\section{Une question de statuts}

Rome a très tôt réfléchi aux possibilités d'alliance entre individus de statuts différents. Pendant longtemps, la « cité patricienne » a refusé toute forme de rapprochement. La loi des XII Tables interdisait tout mariage entre plébéiens et patriciens. Cinq ans à peine après sa promulgation, la lex Canuleia proposée par le tribun de la plèbe $C$. Canuleius lève la prohibition en 445 av. J.-C. Mais l'une des mesures législatives les plus célèbres à traiter de l'éventualité d'une union entre individus de statuts différents s'inscrit dans la série des lois juliennes prises par Auguste entre 18 av. J.-C. et 9 apr. J.-C. Il s'agit de la lex Iulia de maritandis ordinibus, datée de 18 av. J.-C., selon Cassius Dion (Histoire romaine, 56, 7), qui fixe de nouvelles règles en matière matrimoniale. Souvent présentée comme une œuvre de restauration de l'antique mos maiorum, elle interdit, par exemple, le mariage des sénateurs et de leurs enfants jusqu'à trois générations, avec des affranchies - que les chevaliers gardent le droit d'épouser - ou des femmes exerçant des professions jugées incompatibles avec la dignité de l'ordre (prostituées, comédiennes, etc.). Un sénatus-consulte de Claude (52 apr. J.-C.) alourdit même ce régime en interdisant à toutes les femmes citoyennes d'entretenir tout commerce avec un esclave sous peine d'être à leur tour réduites en esclavage. La lex Iulia frappait également d'incapacité testamentaire les célibataires et accordait des avantages aux individus mariés et pourvus d'enfants. Ce dispositif fut sans doute complété par la lex Iulia de adulteriis et de pudicitia qui punissait les conduites jugées scandaleuses tout en durcissant les conditions du divorce et l'adultère, jusque-là passible d'un tribunal domestique, fut requalifié en "crime public $»^{22}$. Il est habituel de présenter la fille d'Auguste, Julie, comme une victime de cette loi et l'intransigeance de son père à la faire revenir d'exil comme la volonté de s'en montrer comme le premier garant (Suétone, Auguste, 65). Enfin en 9 apr. J.-C, fut votée une troisième loi, qui sert d'amendement aux précédentes, la lex Papia Poppaea, du nom des deux consuls en charge, M. Papius Mutilius et $Q$. Poppaeus Secundus, qui la proposèrent. 
Elle est notamment connue par Gaius (Institutes, 1, 178) et par Cassius Dion (Histoire romaine, 56,10$)$ qui, pour mieux insister peut-être sur le rôle de commanditaire tenu par Auguste, glisse - perfidement dit-on - que les deux consuls étaient restés célibataires. Cette nouvelle réglementation reprend en partie le contenu des précédentes lois caducaires, sanctionnant les célibataires et les couples demeurés sans enfant par des mesures limitant leurs capacités à hériter ou à tester. La loi fait notamment bénéficier les couples mariés prolifiques du droit des enfants (ius liberorum), dont les modalités d'application font l'objet dans ce dossier d'une étude détaillée de Sabine Armani. Elle est également connue par un certain nombre de clauses qui fixaient la succession des affranchis et la part revenant aux patrons et aux patronnes subordonnée au nombre de leurs enfants respectifs. Cette loi est souvent décrite comme nataliste. Il est incontestable que le nombre d'enfants sert de critère dans un certain nombre des dispositions décrites. Cependant, le seuil des enfants à atteindre varie en fonction des situations et surtout des statuts et s'avère même inversement proportionnel à la qualité du statut des parents. Ainsi, dans la plupart des cas, trois enfants suffiront à la mère ingénue, là où il en faudra parfois quatre à la mère affranchie pour être dégagée de la tutelle de son patron ou de sa patronne, voire cinq à la mère esclave pour éventuellement être émancipée. La natalité n'était donc pas le critère déterminant, en tout cas pour les femmes citoyennes et ingénues ${ }^{23}$. La lex Papia Poppaea a connu dernièrement un regain d'intérêt suscité par la découverte et la diffusion dans la communauté scientifique, dans des conditions assez extravagantes ${ }^{24}$, d'une nouvelle constitution municipale. Il s'agit du document connu maintenant sous le nom de lex municipalis Troesmensium (loi municipale de Troesmis), en provenance du municipe de Mésie Inférieure, fondé entre 177 et $180 \mathrm{apr}$. J.-C ${ }^{25}$. La loi se présentait matériellement sous la forme de deux grandes tables (appelées par les éditeurs Table A et Table B) dont le contenu est partiellement connu par les deux fragments de bronze découverts. Si la Table A comprenait une partie d'un chapitre (le onzième) traitant des ambassades municipales, qui reprend en partie, mais en le développant davantage, le paragraphe 45 de la lex Irnitana, en revanche, la table B dont il reste la fin du chapitre XXVII et le début du chapitre suivant, évoque les conditions d'éligibilité des magistrats dans le municipe. Des passages font écho à des critères déjà notifiés dans les constitutions hispaniques, mais aussi aux réglementations de la lex Papia Poppaea qui fixait l'âge de candidature aux magistratures et aurait permis d'abaisser l'âge d'un candidat à moins de vingt-cinq ans pourvu qu'il ait déjà deux enfants non émancipés. Le réglement de Troesmis nous apprend que la loi de 9 apr. J.-C. (abrégée dans le texte en P (apia) P (oppaea)) et la lex Iulia de 18 av. J.-C. auraient compris à elles deux dix-neuf chapitres de plus que les trente-cinq évoqués par le Digeste $(23,2,19)$. La constitution municipale se fonde sur le contenu d'un commentarius publié le 28 juin 5 apr. J.-C. qui aurait constitué la base de travail à partir de laquelle la lex Papia aurait été votée quatre ans plus tard. Selon W. Eck, ce décalage chronologique traduirait les réticences suscitées dans l'aristocratie par la lex Iulia de 18 av. J.-C. concernant le mariage, la procréation et le divorce. Auguste aurait alors accordé un moratoire aux célibataires le temps de se mettre en conformité avec la nouvelle loi. Un premier délai entre la première loi et son application, évoqué par les sources littéraires (Cassius Dion, Histoire romaine, 56, 7) correspondrait à la publication du commentarius, resté, en raison de la conjoncture défavorable, sans effet jusqu'au vote de la lex Papia $^{26}$. 


\section{Le dispositif législatif garant de la transmission en ligne patrilinéaire}

24 Les lois matrimoniales ne concernaient que les citoyens romains ingénus ou d'origine affranchie. Mais l'absorption de l'Italie et des régions ultramarines avait également mis sur la route de Rome d'autres peuples pour lesquels fut progressivement conçu un dispositif législatif assurant la vie en communauté ${ }^{27}$. Pour les populations restées en dehors du droit latin et donc privées du ius conubii qui lui était associé, il était impossible de conclure de justes noces avec un citoyen romain. C'est pourquoi, Tite-Live (Histoire romaine, 43,3 ) évoquant les enfants nés d'unions illégitimes entre vétérans italiques et femmes originaires de la péninsule Ibérique, parle d'hommes d'un nouveau genre (noui generis). Ces enfants, exclus de la succession légale, avaient envoyé une ambassade au sénat pour obtenir une forme de reconnaissance. L'historiographie la plus récente vient de chasser une idée reçue concernant l'intervention des autorités impériales dans la vie $\mathrm{du}$ soldat romain. L'autorisation donnée aux légionnaires de se marier pendant leur service - mesure que l'on associait d'autant plus facilement aux Sévères que la « dynastie » était qualifiée de «militaire »- a été remise en question sur la base d'une relecture d'Hérodien ${ }^{28}$. La diffusion du droit latin aidant, on avait aussi avancé un temps l'hypothèse, fondée sur une interprétation erronée de transmission du gentilice maternel aux enfants en contexte provincial, d'une réforme d'Hadrien qui aurait étendu aux enfants nés d'un père pérégrin et d'une mère citoyenne romaine, le privilège de la citoyenneté romaine, ce qui revenait à dire que les citoyennes romaines jouissaient dès lors du ius conubii ${ }^{29}$. Les sources épigraphiques n'ont pas permis d'étayer cette thèse contraire de toute façon aux principes de nature agnatique d'organisation de la famille romaine qui assure la transmission du nom en ligne paternelle.

Garantir l'exercice de la patria potestas et de certains de ses pouvoirs exorbitants laissés au père de famille fut l'un des principaux soucis des juristes. Vient bien sûr à l'esprit le droit de vie et de mort (ius uitae necisque) du père sur ses enfants, dont l'exemple archétypal et fondateur reste celui de Brutus en 509 av. J.-C. Dans ce dossier, Philippe Moreau réévalue d'ailleurs, avec de nouveaux arguments, l'historicité d'une loi de Romulus concernant la première-née.

Mais l'un des effets inattendus de la patria potestas est qu'elle s'étend aussi aux enfants posthumes puisque son application est la «conséquence directe de la naissance ${ }^{30}$ ». Un enfant à naître, qu'un père aurait oublié d'exhéréder depuis la loi Iunia Vellea de 28 apr. J.-C., était protégé en tant qu'héritier présomptif de son père, au nom de l'absence de rupture de la puissance paternelle. En l'absence de son mari, la femme enceinte était dotée de curateurs au ventre, chargés de veiller sur les droits de l'enfant in utero et sur celui du père, mort ou vif ${ }^{31}$. Une femme enceinte de son mari prisonnier de l'ennemi devait demeurer auprès de son beau-père qui détenait la patria potestas sur sa descendance. Enfin, même s'il existait de nombreuses exceptions en raison des configurations différentes des familles ${ }^{32}$, un enfant dont le père était mort ou divorcé, ne devait pas "couper les ponts" avec sa famille paternelle seule garante de ses droits successoraux et de ceux de son père, sans qu'il y ait aucune prescription juridique à ce sujet. C'est ce qu'il faut déduire de l'anecdote ironique rapportée par Suétone, à propos de l'éducation du jeune Néron. Le biographe écrit qu'elle fut confiée à sa tante paternelle qui, au lieu de lui inculquer les bonnes mœurs, lui apprit la débauche (Suétone, Néron, 5)! 
C'est pourquoi aussi, l'« enlèvement » de Livie par Auguste, alors qu'elle était enceinte de son premier mari, allait à l'encontre des codes aristocratiques romains (Suétone, Auguste, 62). Y. Thomas relève qu'un rescrit impérial d'Hadrien (Ulpien, Digeste, $1,5,18$ ) empêchait l'exécution des femmes enceintes jusqu'à la délivrance :

L'empereur Hadrien a répondu par lettre à Publius Marcellus que si une femme libre est condamnée à la peine de mort étant enceinte, l'enfant qu'elle met au monde est libre, et qu'il est habituel d'épargner la vie de cette femme jusqu'au moment où elle a mis l'enfant au monde ${ }^{33}$.

Selon le juriste, c'est la capacité du père à avoir un héritier légitime qui est ainsi préservée. La promesse contenue dans la naissance était telle que Postumus servit de cognomen au fils posthume d'Agrippa et Julie, pressenti un temps pour succéder à Auguste. C'est dans cet esprit aussi que fut conçu, sous Septime Sévère, le premier rescrit à condamner l'avortement sans le consentement de l'ex-époux (Digeste, 47, 11, 4 et 48, 19, $39)^{34}$. À cet égard, on fera observer que le crime d'avortement ne concernait que les femmes mariées ${ }^{35}$. La protection des biens du père et sa capacité à avoir un héritier s'expriment donc aussi dans le délai de viduité imposée aux veuves afin qu'un enfant conçu, mais non encore né, ne voie pas le jour dans une autre maison et sous une autre puissance paternelle. De ce principe, découle aussi l'application systématique de la formule juridique : «pater est quem nuptiae demonstrant » en cas de doute sur la paternité ( Digeste, 2, 4, 5). Libre ensuite au père "social» de faire exposer l'enfant soupçonné d'illégitimité. Ces différents exemples réaffirment le fondement du mariage qui réside dans la procréation des enfants ${ }^{36}$. C'est pourquoi, les capacités physiologiques des individus sont prises différemment en compte selon le législateur et les impératifs du moment. Ainsi un sénatus-consulte de Tibère durcit un temps les conditions de la lex Papia Poppaea qui libérait les femmes de plus de 50 ans et les hommes de plus de 60 ans des obligations matrimoniales et du devoir de procréation. En 34 apr. J.-C. le sénatus-consulte Pernicianum ou Persicianum prescrivait que dans les cas de mariages contractés par un individu de plus de 60 ans avec un conjoint de moins de 50 ans ou l'inverse, les époux seraient frappés d'incapacité testamentaire ou d'héritage jusqu'à ce que Claude assouplisse le régime (Suétone, Claude, 23, 3) 37 .

\section{Préserver le « bon ordre du monde »}

Avec les alumni, Rome n'a pas ni tout à fait l'équivalent des threptoi (enfants nourriciers de statut servile) ni des trophimoi (qui, eux, sont ingénus) de la Grèce ancienne ${ }^{38}$. Le mot alumnus, dont l'étymologie formée sur alere (nourrir en latin) pourrait certes avoir valeur de correspondance avec le verbe grec trephein, désigne une catégorie moins explicite d'individus - ingénus ou non - qui, outre les nourrissons, sont liés, par une « relation quasi-familiale ${ }^{39}$ » et normalement indéfectible, avec les personnes qui ont assuré leur entretien. Les esclaves, souvent nés dans la maison du maitre, ont alors vocation à devenir leurs affranchis. Une expression épigraphique bien étudiée ${ }^{40}$ - l'alumnus ciuitatis pourrait être un lointain écho de cette pratique. La formule attestée seulement dans les cités d'Afrique a été rendue par la traduction moderne « enfant du pays » qui ne rend pas bien compte toutefois de la dimension nourricière, pourtant au cœur des enjeux de la vie quotidienne.

29 À Rome cependant, la solidaritét ${ }^{41}$ envers les indigents n'était pas conçue de manière institutionnelle et était le plus souvent laissée aux familles - qui avaient par exemple le 
devoir de doter les parentes dans le besoin - et aux cités. Cependant, tous les habitants n'étaient pas forcément conviés à la table des bienfaiteurs. Des expressions épigraphiques suggèrent que les femmes n'étaient pas nécessairement invitées ${ }^{42}$ ni même tous les groupes d'âge. La question peut également se poser pour les incolae voire les simples résidents.

Loin d'être réservées aux plus démunis, les distributions alimentaires (frumentationes) étaient l'apanage de ce que d'aucuns appellent commodément les "classes moyennes». N'étaient éligibles à ces distributions de cinq modii de blé par mois (l'équivalent d'environ $35 \mathrm{~kg}$ ), à titre individuel et non héréditaire, que les citoyens mâles et adultes, habitants ou originaires de Rome et inscrits, par tirage au sort, sur les rôles de la plèbe frumentaire qui représentait entre 150000 et 200000 personnes selon les époques ${ }^{43}$. En étaient vraisemblablement exclus les affranchis, sauf en cas de procédure spéciale d'admission, comme certains membres de collèges aux activités reconnues d'utilité publique ${ }^{44}$. Les fillettes, également tenues à l'écart de ces distributions, pouvaient à certaines conditions bénéficier, comme les jeunes garçons inscrits sur les listes, des alimenta impériaux, de fondations alimentaires particulières, comme celles instituées par certains Antonins à l'occasion du décès d'une impératrice, fondations connues sous le nom de "puellae

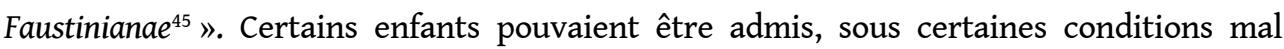
élucidées, à des distributions exceptionnelles en nature, auxquelles pouvaient procéder les empereurs: les congiaires. Ces distributions étaient peut-être censées faciliter l'intégration de nouveaux-venus et leur enrôlement dans l'armée.

Depuis toujours, en effet, les soldats avaient fait l'objet de prévenances particulières. Sous la République, ils avaient pu bénéficier, avec des civils, d'installations dans les provinces. Auguste mit en place, à destination des vétérans, la uicesima hereditatium, un impôt prélevé sur les héritages, qui servait à financer leur « honesta missio » (Suétone, Auguste, 49), c'est-à-dire leur congé définitif.

Les effets de la patria postestas devaient en principe suffire à assurer les vieux jours du chef de famille. Dans le cas contraire, on attendait que les enfants prennent en charge leurs vieux parents ${ }^{46}$. L'épigraphie suggère que la solidarité devait en général bien s'exercer puisque la prédominance de la famille nucléaire dans les inscriptions ${ }^{47}$ s'explique en grande partie par le devoir de pietas ${ }^{48}$ des descendants. La réciproque n'était pas toujours vraie puisqu'un rescrit d'Antonin le Pieux décide que :

Des juges compétents saisis par toi ordonneront que tu sois nourri par ton père selon ses ressources, pourvu que, étant un travailleur, tu sois dans un état de santé qui t'empêche de satisfaire aux tâches (Digeste, 25, 3, 5, 7) 49. $^{4}$.

Cependant, sous le règne suivant de Marc Aurèle et de Lucius Verus, une constitution fait droit aux réclamations d'un père :

Un juge compétent donnera l'ordre que tu sois entretenu par ton fils, s'il en a la possibilité, pour que ta nourriture soit assurée (Code Justinien, V, 25, 2).

Les torts devaient souvent être partagés puisque d'autres constitutions de la même époque rappellent l'obligation réciproque d'assistance alimentaire, mais leur réitération indique la difficulté de son application. Il faut néanmoins attendre 374 apr. J.-C. pour qu'une décision impériale oblige les parents à élever leurs enfants ${ }^{50}$. Malgré le titre de pater patriae porté par le Princeps dès Auguste, les interventions impériales en la matière sont donc relativement tardives et ne sont pas attestées avant le II $^{\mathrm{e}}$ siècle apr. J.-C., époque où la notion de securitas assurée par l'empereur se développe ${ }^{51}$. Enfin, les préoccupations hygiénistes, permettant d'assurer la survie des enfants, n'étaient pas 
complètement absentes de la réflexion des Anciens. L'allaitement est même recommandé, mais ne sied pas aux femmes de l'aristocratie qui préfèrent faire appel à une nourrice. Soranos d'Ephèse, qui exerça à Rome au $\mathrm{II}^{\mathrm{e}} \mathrm{s}$. apr. J.-C., préconisait par exemple l'emmaillotement des enfants, sans que l'on puisse mesurer l'effet réel de ces conseils.

D'ailleurs, cette même période voit se dessiner une évolution de la patria potestas en faveur d'une solidarité plus grande entre générations que l'on a mise sur le compte d'une transformation des mœurs. Si Auguste réaffirma le droit d'un père à prononcer une sentence de mort (ius uitae necisque) contre son fils venant ainsi à l'aide du chevalier Trico lapidé par la foule pour avoir prononcé un verdict de ce type (Sénèque, de clementia, 1, 15), Hadrien punit un père qui avait tué son fils coupable d'adultère avec sa belle-mère (Marcien, Digeste, 48, 9, 5) ${ }^{52}$. À l'époque de Justinien, les législateurs parlaient désormais de la puissance paternelle au passé.

La bonne marche de la cité et de l'empire est également symboliquement assurée par le renouvellement des générations et le respect de leur enchaînement ${ }^{53}$. De la République à l'Empire, on a des traces régulières d'assouplissement de la législation matrimoniale en matière d'interdits touchant les collatéraux avant d'assister à un mouvement inverse à partir de Dioclétien, c'est-à-dire bien avant que ne s'impose le christianisme. Comme exemple de levée de prohibition matrimoniale, on n'évoquera qu'un seul cas, sans doute le plus célèbre. Il s'agit de l'autorisation votée par le sénat du mariage normalement frappé de l'accusation d'inceste de l'empereur Claude et de sa nièce (fille de son frère), Agrippine :

Il soudoya des sénateurs qui, à la première séance du sénat, proposèrent qu'on le contraignît à l'épouser, soi-disant dans l'intérêt supérieur de l'État, et qu'on autorisât tous les citoyens à contracter de pareilles unions, regardées jusqu'alors comme incestueuses. Puis, après un intervalle d'un jour à peine, il célébra le mariage, mais il ne trouva personne pour suivre son exemple, si ce n'est un affranchi et un centurion primipilaire, aux noces duquel il assista lui-même, en compagnie d'Agrippine (Suétone, Claude, 26).

Malgré les différences constatées entre le monde grec, le monde romain et au-delà, la réflexion suscitée par l'étude des "politiques familiales" doit contribuer à jeter un regard éclairant sur les liens entre pouvoir, famille et sociétés. Les spécificités repérées à Rome, à Gortyne, à Sparte ou encore à Athènes façonnent finalement autant de modèles que de cités, avec parfois des dénominateurs communs, dessinant une mosaïque de systèmes sociaux et familiaux. Une richesse et des nuances que les candidats et les préparateurs aux concours 2018-2019 de l'agrégation d'histoire et du capes d'histoiregéographie pourront apprécier par le biais de la question «Famille et société dans le monde grec et en Italie ( $\mathrm{v}^{\mathrm{e}}-\mathrm{II}^{\mathrm{e}}$ siècles av. J.-C.) ».

BIBLIOGRAPHIE

Adam-Magnissali S. (2007), « Droit et altérité dans le monde ancien : le cas des mineurs dans l'Athènes classique », Symposion 20, p. 145-160. 
Armani S. (2010), «Les pérégrins dans les cités romaines de Lusitanie aux deux premiers siècles de notre ère : identités et pratiques onomastiques », dans Gorges J.-G. et Nogales Basarrate T. éd., $\mathrm{VII}^{e}$ table ronde internationale sur la Lusitanie romaine "Naissance de la Lusitanie romaine (I ${ }^{\text {er }}$ av. - I ${ }^{\text {r }}$ ap. J.-C.)/Origen de la Lusitania romana (siglos I a. C.-I d. C.), Toulouse-Mérida, p. 293-318.

Avotins I. (2004), « Athenaion politeia 56.6 and the protection of the weak », CQ 54-2, p. 461-469.

Bernard N. (2017), « Nourrir les vieux. De l'oikos à la cité », dans Guilhembet J.-P. et al. éd., Famille et société dans le monde grec et en Italie du Ve siècle au II siècle av. J.-C., Paris, p. 296-305.

Chastagnol A. (1998), « La condition des enfants issus de mariages inégaux entre citoyens romains et pérégrines dans les cités provinciales de droit latin », dans Epigrafia romana in area adriatica. IXe Rencontre franco-italienne sur l'épigraphie du monde romain, Macerata, 10-11 novembre 1995, Pise-Rome, p. 249-262.

Corbier M. (1990a), « Usages publics et vocabulaire de la parenté : patronus et alumnus de la cité dans l'Afrique romaine ", dans Mastino A. éd., L'Africa romana. Atti del VII Convegno di studio, Sassari, 15-15 dicembre 1989, Sassari, p. 815-854.

- (1990b), « Alumnus ou alumna de la cité : un hommage des cités d'Afrique à leurs notables et bienfaiteurs », BSAF, p. 323-326.

- (1998), «Épigraphie et parenté », dans Le Bohec Y. et Roman Y. éd., Épigraphie et histoire : acquis et problèmes. Actes du colloque de la SoPHAU, Lyon-Chambéry, 21-23 mai 1993, Lyon, p. 101-152.

- (1999a), « La petite enfance à Rome : lois, normes, pratiques individuelles et collectives », Annales (HSS) 6-4, p. 1257-1290.

- (1999b), « Adoptés et nourris », dans Corbier M. dir., Adoption et fosterage, Paris, p. 5-41.

- (2001), «Solidarité entre les générations et sécurité du cycle de vie : la société romaine (II ${ }^{\mathrm{e}}$ siècle avant J.-C. - III siècle après J.-C.) ", dans Guillaume P. dir., Les solidarités. Le lien social dans tous ses états. Colloque de Bordeaux, 16-17 juin 2000, Bordeaux, p. 103-123.

- (2005), «L'uno e l'altro sesso : epigrafia e frontiera di gender », Epigraphica 67, p. 341-366.

- (2008), « Épigraphie et études de genre », REL 86, p. 152-166.

Damet A. (2012), La septième porte. Les conflits familiaux dans l'Athènes classique, Paris.

- (2017), « De Sparte à l'île du Soleil. Eugénisme et communauté des enfants dans les utopies de la Grèce ancienne », Annales de Bretagne et des Pays de l'Ouest, 124-3, p. 31-48.

Damet A. et Moreau Ph. (2017), Famille et société dans le monde grec et en Italie du V $V^{e}$ s. av. J.-C. au II ${ }^{e} s$. av. J.-C., Paris.

Eck W. (2013), « La loi municipale de Troesmis : données juridiques et politiques d'une inscription récemment découverte », RD 2, p. 199-213.

- (2016), « Die lex municipalis Troesmensium : ihr rechtlicher und politisch-sozialer Kontext », dans Alexandrescu Ch.-G. éd., Troesmis. A Changing Landscape. Romans and the Others in the Lower Danube Region in the First Century BC-Third Century AD. Proceedings of an International Colloquium Tulcea, $7^{\text {th }}-10$ th of October 2015, Cluj-Napoca, p. 33-46.

Flandrin J.-L. (1984), Familles. Parenté, maison, sexualité dans l'ancienne société, Paris.

Fournier J. et Hamon P. (2007), « Les orphelins de guerre de Thasos : un nouveau fragment de la stèle des Braves (ca 360-350 av. J.-C.) », BCH 131-1, p. 309-381.

Gardner J. F. (1986), Women in Roman Law and Society, Londres-Sydney. 
- (1998), Family and Familia in Roman Law and Life, Oxford.

Gascou J. (1999), « Hadrien et le droit latin », ZPE 127, p. 294-300.

Harders A.-C. (2010), « Roman Patchwork Families : Surrogate Parenting, Socialization and the Shapping of Tradition », dans Dasen V. et Späth Th. éd., Children, Memory and Family Identity in Roman Culture, Oxford, p. 49-72.

Inglebert H. dir. (2005), Histoire de la civilisation romaine, Paris.

Kapparis K. (1995), « When were the Athenian adultery laws introduced? », RIDA 42, p. 97-122.

Karabélias K. (2002), L'épiclérat attique, Athènes.

Lajeunesse M. (2012) «Repenser le rapport entre loi et famille dans la Grèce archaïque et classique : l'exemple de la descendance dans les inscriptions à caractère législatif ", Cahiers d'histoire 31-2, p. 181-205.

Lenoir R. (2007), « Politiques familiales et modes de gestion des populations. Dans une perspective historique », Informations sociales 139-3, p. 10-21.

Le Roux P. (2015), « Le droit latin (ius Latii) : une relecture », dans Cresci Marrone G. éd., Trans Padum usque ad Alpes. Roma tra il Po e le Alpi : dalla romanizzazione alla romanità. Atti del convegno, Venise, 13-15 mai 2014, Rome, p. 179-195.

Martin C. (2011), « Enjeux des politiques de la famille en France », Revue Projet 322-3, p. 45-51.

Moreau Ph. (1990), « Adfinitas. La parenté par alliance dans la société romaine ( ${ }^{\mathrm{er}}$ siècle av. J.-C. II ${ }^{\mathrm{e}}$ siècle ap. J.-C.) », dans Andreau J. et Bruhns H. éd., Parenté et stratégies familiales dans l'Antiquité romaine. Actes de la table ronde des 2-4 octobre 1986 (Paris, Maison des sciences de l'homme), Rome, p. 3-26.

Moreau Ph. (2002), Incestus et prohibitae nuptiae. L'inceste à Rome, Paris.

Ogden D. (1996), Greek bastardy in the classical and Hellenistic periods, Oxford.

Oulhen J. (2004), « La société athénienne », dans Brulé P. et Descat R. éd., Le monde grec aux temps classiques, Tome 2, Le IV ${ }^{e}$ siècle, Paris, p. 251-351.

Rémy B. et Mathieu N. (2015), Les vieux en Gaule romaine, Arles.

Roubineau J.-M. (2012), « La cité égoïste ? cité athénienne et action sociale », dans Konuk K. éd., Stephanèphoros. De l'économie antique à l'Asie mineure. Hommages à Raymond Descat, Bordeaux, p. $165-178$.

- (2015), Les cités grecques (VI ${ }^{e}-I^{e}$ siècle av. J.-C.). Essai d'histoire sociale, Paris.

Roux O. (2009), « Parenté hippocratique et parenté aristotélicienne : quelques réflexions sur les théories biologiques de la Grèce ancienne », Pallas 79, p. 307-322.

Roy J. (1999), « 'Polis' and 'Oikos' in Classical Athens », Greece \& Rome 46-1, p. 1-18.

Rubinstein L. (1993), Adoption in IV. century Athens, Copenhague.

Ruzé F. (2006), « En Grèce archaïque : la législation au secours des plus faibles » dans Molin M. éd., Les régulations sociales dans l'Antiquité, Rennes, p. 171-188.

Saller R. P. (1984), « Familia, domus and the Roman conception of the family », Phoenix 38, p. 336-355.

- (1989), «I rapporti di parentela e l'organizzazione familiare », dans Gabba E. et Schiavone A. éd., Storia di Roma, vol. IV (Caratteri e morfologie), Turin, p. 515-555. 
Saller R. P. et Shaw B. D. (1984), « Tombstones and Roman family relationships in the Principate : civilians, soldiers and slaves ", JRS 74, p. 124-156.

Slater N. (1993), « Theozotides on adopted sons: (Lysias fr. 6) », Scholia 2, p. 81-85.

Speidel A. (2013), « Les femmes et la bureaucratie. Quelques réflexions sur l'interdiction du mariage dans l'armée romaine », CCG XXIV, p. 205-215.

Stroud R. S. (1971), « Greek inscriptions. Theozotides and the Athenian orphans », Hesperia 40, p. 280-301.

Thoenig J.-C. (2014), « Politique publique », dans Dictionnaire des politiques publiques, Paris, p. $420-427$.

Thomas Y. (1986), « Le « ventre ». Corps maternel, droit paternel », Le Genre humain 14, p. 211-236.

- (2007), «L'enfant à naître et l'héritier sien. Sujet de pouvoir et sujet de vie en droit romain ", Annales (HSS) 62-1, p. 29-68.

- (2017), La mort du père. Sur le crime de parricide à Rome, Paris.

van Effenterre H. et Ruzé F. (1994-1995), Nomima : recueil d'inscriptions politiques et juridiques de l'archaïsme grec, 2 vol., Rome (= Nomima).

Virlouvet C. (2009), La plèbe frumentaire dans les témoignages épigraphiques. Essai d'histoire sociale et administrative du peuple de Rome antique, Rome.

\section{NOTES}

1. L'œuvre législative d'Aristote, dans ses Politiques, est ainsi pétrie de considérations physiologiques et biologiques (1334b-1335b). Voir, par exemple: «Donc, puisque le législateur doit dès le début surveiller que les enfants aient le meilleur corps possible, il lui faut d'abord se soucier des unions : quand et entre quelles sortes de gens doivent avoir lieu les rapports conjugaux. [...] De plus, pour en revenir à notre point de départ, il faut veiller à ce que le corps des enfants procréés soit conforme à la volonté du législateur ». Voir aussi Roux 2009 sur l'arrière-plan « médical » et « biologique » de la loi de Périclès de 451, à savoir la concurrence entre les différentes théories de la reproduction et leurs liens possibles avec les deux idéologies co-existantes, patrilinéaire ou bilatérale.

2. Voir la chronologie de la politique de la famille en France sur le site de la DILA (Direction de l'information légale et administrative), dans la rubrique « La vie publique ». URL : http://www.vie-publique.fr/politiques-publiques/famille/chronologie/

3. Voir aussi la contribution d'Aurélie Damet dans ce volume.

4. Aristophane, Les Nuées, v. 1068-1083; [Démosthène], LXIX, Contre Nééra, 85-87; Démosthène, Contre Aristocrate, 53 ; Eschine, I, Contre Timarque, 183. Voir Kapparis 1995 ; Hoffman 1996 ; Damet 2012, p. 235-240 ; Damet et Moreau 2017, p. 241-244. Une partie des dispositions sur la répression de la moicheia auraient été introduites à la suite de la loi de Périclès sur la citoyenneté, notamment celles concernant les épouses infidèles, qui risquent d'introduire des nothoi et des nothai dans l'oikos.

5. Sur le divorce dans les lois de Charondas, voir Diodore de Sicile, XII, 18, 1. Sur le divorce à Athènes, voir Andocide, Contre Alcibiade, 14; Lysias, Contre Alcibiade, 28 ; Isée, Succession de Ménéklès, 7-12 ; Succession de Pyrrhos, 78 ; Démosthène, Contre Onètor, 4 ; Contre 
Spoudias, 4 ; Contre Euboulidès, 41 ; Contre Nééra, 51 et 63. Sur le divorce à Gortyne voir Nomima, II, 30. En dernier lieu, voir Damet et Moreau 2017, p. 114-117.

6. Thucydide, II, 54, 3 : «Cependant, il faut être fermes, ne serait-ce que par l'espoir d'autres enfants, si vous êtes encore en âge d'en avoir. Individuellement, leur venue apportera à certains l'oubli de ceux qui ne sont plus, et la cité, elle, en tirera un double avantage, en évitant de se dépeupler, et pour le soin de sa sécurité. »

7. À Rhodes, en 305, suite au siège de Démétrios Poliorcète, la cité met en place le système suivant d'aide aux orphelin(e)s : «Ils rédigèrent un décret selon lequel les hommes morts au combat seraient ensevelis à frais publics, tandis que leurs parents et leurs enfants seraient entretenus et recevraient une aide versée sur la caisse publique : par ailleurs, les filles seraient dotées par les soins de la cité et l'on octroierait au théâtre, lors des Dionysies, une panoplie à leurs fils, une fois arrivés à l'âge adulte » (Diodore de Sicile, XX, 84).

8. Aristote, Politiques, $1268 \mathrm{a} 8$.

9. Sur la cité nourricière et, plus généralement, sur l'idéologie de la «parenté de substitution », voir Démosthène, Oraison funèbre, 36-37 ; Platon, Ménéxène, 248d-249c.

10. Plus exactement, sous l'archontat d'Euclide, Nikomenes aurait proposé qu'un enfant né après 403/402 ne puisse avoir la citoyenneté si ses parents ne sont pas citoyens, mais sans effet rétroactif (scholie à Eschine, Contre Timarque, 39. Voir Isée, Succession de Kiron, 43 et Démosthène, Contre Euboulidès, 30). Aristophon aurait, lui, proposé que les enfants d'un citoyen et d'une mère étrangère soient bien catégorisés comme nothoi (Athénée, XIII, 577b-c ; voir Isée, Succession de Philoktémon, 47). Voir Ogden 1996, p. 76-78.

11. Ps.-Aristote, Constitution d'Athènes, 35, 2: «Ils enlevèrent de l'Aréopage les lois d'Éphialte et d'Archestratos concernant les Aréopagites, et celles des lois de Solon qui provoquaient des discussions, ainsi que le pouvoir de décision souveraine qu'avaient les juges [...]. Par exemple, en ce qui concerne les donations, ils rendirent chacun absolument libre de donner à qui il voudrait et enlevèrent les entraves mises à ce droit: "excepté en état de folie ou de sénilité ou sous l'influence d'une femme", cela afin d'enlever tout moyen d'action aux sycophantes. "

12. Ps.-Aristote, Constitution d'Athènes, 56, 7 ; Démosthène, Contre Macartatos, 75 ; Lysias, Contre Diogiton, 23.

13. Karabélias 2002, p. 197. Sur le pouvoir de l'archonte éponyme comme protecteur des orphelins et des filles épiclères, cf. Lysias, Au sujet de l'examen d'Evandros, 12 ; Démosthène, Contre Lacritos, 48 ; Contre Panténètos, 46 ; Contre Stephanos II, 22 ; Eschine, Contre Timarque, 158.

14. Karabélias 2002, p. 199, n. 4 ; voir L. Gernet, Démosthène, CUF, t. 11, p. 121, n. 4.

15. Voir la stèle des Braves de Thasos (Fournier et Hamon 2007) et le décret de Rhodes, dans Diodore de Sicile, XX, 84, 3 (voir note 7).

16. Voir aussi Ruzé 2006, p. 173 : «Protéger la famille et la femme, pourquoi ? La première réponse est le souci de l'équilibre de la cité par le maintien des cellules de base que sont les familles et leur patrimoine. Une deuxième réponse, très fréquente, est que l'on veut encadrer la femme, trop facilement incontrôlable. Mais je crois que nous sommes trop enclins à nous arrêter à une interprétation misogyne de la législation grecque, tant est ancrée en nous l'idée que la femme, nécessaire à la procréation, était par ailleurs traitée en mineure, que l'on se méfiait d'elle, bref, qu'on ne l'aimait pas. Les psychologues ont peut-être raison de voir dans cette "peur de la femme" exprimée par Hésiode le désarroi 
masculin face à des êtres qu'ils ne peuvent bien comprendre ni maîtriser. Mais cela échappe à la loi. Celle-ci, en revanche, me paraît témoigner bien souvent du souci d'aider la femme dans sa faiblesse, de la protéger, tout en la contrôlant, bien entendu. Cela rejoignait, du reste, l'intérêt bien compris de la communauté, soucieuse de la stabilité des foyers. "

17. Nomima II, 51.

18. Sur ces débats, voir par exemple, Gardner 1998. L'ambiguïé sémantique, relevée pour la langue française, se rencontre dans de nombreuses autres langues modernes.

19. J.-L. Flandrin (1984, p. 11) cite une définition proche pour la famille actuelle : « Mais il y a aussi un sens étroit, beaucoup plus habituel, que les dictionnaires donnent en premier et que les sociologues prennent généralement seul en considération. En ce sens le mot désigne "les personnes apparentées vivant sous le même toit", et plus spécialement le père, la mère et les enfants » (Petit Robert).

20. «Quod ius proprium ciuium Romanorum est » (Ce droit est propre aux citoyens Romains).

21. Les femmes ne faisaient pas partie de la plèbe frumentaire : la seule attestation connue d'une femme - ILS 9275 - est interprétée comme l'exemple d'une veuve ayant charge d'enfant mineur, dont la présence était nécessaire lors de la distribution: voir Virlouvet 2009, p. 247-256, $\mathrm{n}^{\circ} 20$ du catalogue. Mais une absence féminine complète des distributions alimentaires doit être nuancée à l'aune de la documentation : il existait des distributions réservées aux petites filles issues de fondations impériales "genrées" comme les puellae Faustinianae créées par Marc Aurèle en mémoire de sa femme Faustine la Jeune (voir infra). En outre, les cités d'Italie ont révélé l'association de groupes de femmes aux banquets (CIL, XIV, 2120) ou aux distributions d'argent ou de nourriture ( $A E$, 2000, 531 et 533). La sociabilité plus limitée de ces communautés marquées par «l'entre-soi» a été avancée comme argument anthropologique pour expliquer l'association du sexe féminin à ce type d'activités (Corbier 2008).

22. Inglebert 2005, p. 136.

23. Voir infra le dossier consacré au ius trium liberorum.

24. Eck 2013, p. 199 : «En 2003, je reçus de la part d'un de mes correspondants un e-mail, auquel était attachée la version PDF d'un fax qu'il avait reçu d'Angleterre.» et p. 200 : "On pouvait présumer que le texte de cette loi se trouvait sur des tables de bronze, mais on n'avait aucune information à ce sujet. Deux ans plus tard, en 2005, cette incertitude fut levée: car deux grandes tables de bronze, sur lesquelles figurait le texte que je connaissais déjà, furent mises en vente sur eBay... »

25. $A E, 2013,1345$.

26. Eck 2013, p. 204-209 ; Eck 2016, p. 39-41.

27. Le Roux 2015.

28. Speidel 2013.

29. Chastagnol 1995. Contra Gascou 1999 ; Armani 2010, p. 306-308.

30. Corbier 1999a, p. 1264.

31. Thomas 1986, p. $212=$ Thomas 2017, p. 97 .

32. Harders 2010.

33. Thomas 2007, p. $34=$ Thomas 2017, p. 125-126. 
34. «Il est indigne de laisser paraître qu'elle puisse avoir privé son mari d'enfants, impunément et par fraude.» et "Celle qui porte atteinte à ses entrailles après son divorce, parce qu'elle était enceinte, pour ne pas donner de fils à son mari devenu à ce point son ennemi » dans Thomas 2007, p. 34.

35. Gardner 1986, p. 139.

36. Moreau 1990, p. 3 : « Pourquoi se marier ? "Pour acquérir une descendance légitime", répond avec constance, la tradition romaine, liberum quaesundorum causa : cette formule, attestée depuis le II $^{\mathrm{e}}$ siècle av. J.-C. chez Ennius et Plaute, figurait dans le formulaire officiel du recensement quinquennal et on l'inscrivait dans les actes privés, les contrats de mariage. »

37. Voir aussi Rémy et Mathieu 2015, p. 113.

38. Corbier 1999a, p. 1283.

39. Corbier 1999b, p. 28.

40. Corbier 1990a et 1990b.

41. À propos de ce terme, Mireille Corbier (2001, p. 105) fait observer que la notion moderne de solidarité a été introduite tardivement en français. Le mot tire son étymologie du vocable latin solidus qui désigne le lien qui unit les débiteurs d'une somme entre eux. Ce sens demeure en droit. Mais la dimension de responsabilité morale et mutuelle qu'on lui prête aujourd'hui était absente de la notion d'origine. En revanche, le latin était doté d'une série de termes fondés sur une conception hiérarchique des relations sociales (necessitudo, officium, etc.) visant à identifier les devoirs réciproques, mais à géométrie variable, qui liaient entre eux les parents, les amis ou les subordonnés à leurs patrons.

42. Corbier 2005. Elles n'étaient pas non plus systématiquement exclues (voir supra).

43. Virlouvet 2009.

44. Virlouvet 2009, p. 61-68.

45. Virlouvet 2009, p. 69-73.

46. CIL, XIII, 2036 déplore le décès prématuré d'un jeune homme en qui son beau-père ( uitricus) « avait placé l'espérance de ses vieux ans » (traduction de Corbier 2001, p. 111).

47. Saller et Shaw 1984, p. 124-156.

48. Corbier 1998, p. 102.

49. Cité par Rémy et Mathieu 2015, p. 126.

50. Corbier 1999a, p. 1265 : «Que chacun nourrisse sa progéniture.»

51. Pline le Jeune, Panégyrique de Trajan, 27, 1.

52. Les deux exemples sont cités par Saller 1989, p. 534.

53. Moreau 2002. 


\section{AUTEURS}

\section{SABINE ARMANI}

Université Paris 13 (Sorbonne-Paris-Cité)

\section{AURÉLIE DAMET}

Université Paris 1 Panthéon-Sorbonne, UMR 8210 ANHIMA 